\title{
Characterization of the mitochondrial Huso huso genome with new aspects into its organization with presence of tandem repeat in the 12SrRNA and tRNA-Glu
}

\section{Khadijeh Dadkhah ( $\sim$ kdadkhah29@gmail.com )}

Sari Agricultural Sciences and Natural Resources University

Ghodrat Rahimi Mianji

Sari Agricultural Sciences and Natural Resources University

Ali barzegar

Sari Agricultural Sciences and Natural Resources University

Ayoub Farhadi

Sari Agricultural Sciences and Natural Resources University

\section{Research Article}

Keywords: Huso huso, mitogenome, sturgeon, Caspian Sea, tandem repeats

Posted Date: September 14th, 2021

DOI: https://doi.org/10.21203/rs.3.rs-894057/v1

License: @ (1) This work is licensed under a Creative Commons Attribution 4.0 International License. Read Full License 


\section{Abstract}

The sturgeon group is an economically important group in the world due to the production of caviar, and they are also a suitable old species for researching on the evolution of the mitochondrial genome. In H.huso sequencing, we identified a distinct genome organization relative to other species that has never been reported before. In this specie, the mitochondrial genome consisted of 13 genes encoding proteins, 22tRNA and 2rRNA, and two non-coding regions that followed other vertebrates. In addition, H.huso had an pseudo tRNA-Glu between ND6 and Cytb and also had a 52-nucleotide tandem repeat with two replications in the 12SrRNA. This duplication event is related to the slipped strand during replication, which can remain in the strand as a result of mispairing during replication. Furthermore, an 82 bp repeat sequence with three replications was observed in the D-loop control region, which is usually visible in different species. Regulatory elements are also visible in the control region of the mitochondrial genome that include termination sequences and conserved regulatory blocks. Genomic compounds showed the highest conservation in terms of rRNA and tRNA, while protein-encoded genes and non-encoded regions had the highest divergence. The mitochondrial genome was phylogenetically assayed using 13 protein-encoding genes.

\section{Introduction}

Studying the molecular genetics of sturgeon can provide a good way to identify their origin and uncertain aspects. Sturgeons consist of two main genera, Huso and Acipenser. Both genera can be found in the Caspian Sea. Most sturgeons have been identified as endangered by the International Union for Conservation of Nature (IUCN) ${ }^{13}$. Researches had shown that caviar production has declined in Europe and North America, as well as in Russia and $\operatorname{Iran}^{43}$.

Molecular studies on the genetic structure can be useful in identifying valuable information about this endangered fish. In determining the genetic structure of Caspian sturgeon, molecular techniques such as RFLP ${ }^{41}$, RAPD ${ }^{51}$, AFLP ${ }^{11,55,56}$, microsatellite ${ }^{1,1718,31,35}$ and DNA sequencing ${ }^{38,8}$ have replaced traditional methods such as meristic and morphometric. DNA sequencing is one of the most accurate methods of identifying information about sturgeon ${ }^{36}$.Mugue et al. (2008) distinguished sturgeon at specie level with mitochondrial D-loop sequencing ${ }^{36}$. In another study, mitochondrial D-loop sequencing was used as a complementary method with other laboratory methods ${ }^{2}$. Mitochondrial genome sequencing, however, is a simple, immediate, and reliable method to identify species. The Cytb and control region have been used mainly in the identification of sturgeon species. But researchers have found that the cytb gene does not have a good resolution for identifying these species ${ }^{36}$. The size of mitochondrial DNA is about $15-20 \mathrm{~kb}$ and the mitochondrial genome contains several copies and is double-stranded and has a circular molecule. It contains 13 protein encoding genes and two genes encoding ribosomes, 22 genes encoding tRNAs, and two control regions. Because mitochondrial genome DNA is inherited from mother ${ }^{27}$, it is suitable for evolutionary and historical studies ${ }^{48}$. Ludwig et al. (2000) observed in some sturgeon species; VNTR duplicate blocks were in the control region in Sect. 5 immediately after proline tRNA, which was 3 replicates with the size of 82 bp $^{29}$. Mugue et al. 2016 also reported a repetitive sequence of 82 pairs of bases in the ship specie ${ }^{37}$. Researchers with investigating the control region, related the formation of duplicate blocks with the genes close to CR (12 SrRNA and tRNA) ${ }^{42}$. Ciftici et al. (2013) by sequencing the D-loop regions in acipenser gueldenstaedtii, acipenser stellatus and huso huso species, noticed the presence of duplicate blocks in this region and reported a size of 82-83 bp tandem repeat ${ }^{7}$. The frequency of multiple copies of the genome in a specie may be attributed to differences in mutation rates or a mechanism that may regulate mutation rates. A large number of studies have been performed on the teleost of control region that identified VNTR $7,37,29$, but in this study we also detected the presence of tandem repeat in the other two regions, 12 SrRNA and tRNA. So far, there have been reports of excess tRNA in several species of fish $3,12,32,53,57,58$, but there have been no reports about the presence of repeats in fish, in 12srRNA.

This study showed that the mitochondrial genome of sturgeon can differ in organization and in gene content and order.

Our interest in this assay was initially the study of the mitochondrial genome and its molecular mechanisms because mtDNA is an important tool for the assessment of evolutionary biology and is very attractive.

Currently, sturgeon species are at risk of damage and require careful genetic testing to

protect natural resources. The present experiment, due to the availability of mitochondrial genome sequences, allow us to take steps in classification as well as new molecular sources in species identification, aquatic management, and the conservation biology of genetic and biological resources. 


\section{Material And Methods}

\subsection{Sample collection and PCR amplification and sequencing}

Samples were prepared from the southern shore of the Caspian Sea (coast of Iran). Species were identified and samples were collected from the caudal fin. Complete genome DNA was obtained using the ammonium acetate method ${ }^{33}$, and using 16 pairs of universal primers overlapping fragments of the mitochondrial genome were amplified and then sequenced.

Primers and PCR reactions were performed based on the described methods shao et al (2014) with slight modifications ${ }^{47}$. The $100 \mathrm{ml}$ of PCR products, along with $50 \mathrm{ml}$ of each forward and reverse primer $(10 \mathrm{pmol})$ were used to determine the sequences of DNA fragments using Sanger method by European company of the Microsynth.

\subsection{Assembling mitochondrial genome sequences and annotating}

The sequencing results were first manually corrected and edited using (chromasprov.1.42), and then the mitochondrial genome was searched for protein and rRNA using the BLAST tool. Http: //www.ncbi.nlm.nil .gov / BLAST.cgi and annotations were performed based on the mitochondrial genome alignment of closely related species in the Gene Bank database.

The obtained sequences from 16 pieces were aligned with the Clustal $X$ and then edited with the BIOEDIT software, respectively.

The overlapping sequences were assembled by using the SeqMan module of the Lasergene 11.0 software (DNASTAR, Madison, WI, USA). The results of the contigs were mapped to reach the complete mitochondrial genome of the Huso huso. Sequence results and their combined annotations were used to map the specie genome. Most tRNA genes and their secondary structures were predictable by tRNA-scan ${ }^{21}$. Clover leaf structure was detected by computer.

\subsection{Sequence analysis}

Nucleotide compositions were obtained using the DNASTAR program and ATskew and GCskew were calculated using the formula ${ }^{40}$.

AT skew $=[A-T] /[A+T]$ and $G C$ skew $=[G-C] /[G+C]$

Repeat Finder tandem was used to identify duplicate sequences. In the complete mitochondrial genome sequencing of Ka / Ks, the ratio of protein coding genes was calculated.

The codon usage of 13 protein coding genes was calculated by using the Mega 10 software.

\subsection{Phylogenetic analysis}

In this study, the mitochondrial genome of 12 sturgeon species was obtained from Gene Bank. We used European, Chinese, Russian and American sturgeon species for phylogeny. Sequences of 13 genes encoding protein were aligned with the GLUSTALX and then Minor manual settings were used.

To compare the differences, probability tests were performed. Different models were used to compare and determine, and the best model selection based on BIC was determined for 13 protein coding genes. Finally, GTR + I + G was selected as the best model and 12 genes based on Maximum likelihood and Gama distribution were evaluated using all sites and Strap boot 1000.

\section{Results And Discussion}

\subsection{Genome Organization Results}

The complete mitogenome of Huso huso was deposited in Gene Bank (Accession number: MK213068). The Huso huso mitochondrial genome contained $16836 \mathrm{bp}$. This genome normally contains 37 encoding genes, 13 genes encoding proteins, 22tRNA and 2rRNA, and two OL and D-loop control regions, in which an additional tandem repeat tRNA- Glu was observed.

Like many mitochondrial genomes, most genes were located in the heavy strand except ND6 and 8 tRNA (TRNAGLN, TRNAala, tRNAGLU, tRNAser, tyr tyr and tRNAcys, tRNA Asn and tRNA Pro). They are coded in the light strand (Figure 1). 
The nucleotide composition of the genome is shown in Table 2.

Nucleotide asymmetry of the strand is usually described by AT and GC skew.

While GCSKEW is barely above zero (they are mostly negative). These results show that the content of $A$ is only slightly higher than $T$ while $\mathrm{C}$ is significantly higher than $\mathrm{G}$. Skews are related to the difference in mutation pressures applied in light and heavy strings ${ }^{20}$.As a result, they are asymmetric and cause such changes in mtDNA 9, 10, 50 .

\subsection{Genome organization and evolutionary mechanism}

A new type of mitochondrial genome organization has been found to contain an additional tRNA. This extra tRNA can be folded, and create a clover leaf structure of tRNA that also contains an anticodon.

This tRNA is very similar to the conventional tRNA sequence and is located between ND6 and Cytb. This is a new genome organization, and it can create new specific features in the genus H.huso that distinguish it from other sturgeon species. Changes in the tRNA of other fish species have also been observed. In a study in a fish specie, generated additional tRNA-lle with anticodon mutations TAC to AAT. It has also been observed in serranidae that the extra tRNA-Asp in the light strand is the result of rearrangement of the mitochondrial genome ${ }^{6,24}$. This gene content of the fish mitochondrial genome is typically the result of gene amplification and causes diversity in species. The rearrangement of the mitochondrial genome observed in fish usually can include trans location ${ }^{23,25}$, which is also due to the tandem duplication of gene regions. This example shows that extra tRNA is left in the mitochondrial genome after changes. Abnormal fish mitochondrial genome tRNAs include extra tRNA-ser at the downstream ND5 in sea bass Morone saxatilis ${ }^{53}$ and extra tRNA met in Pampus species ${ }^{12}$ and the pseudo tRNA at the same position in parrotfish Chlorurus sordidus ${ }^{32}$ and extra tRNA Asn and pseudo tRNA ala in the WANCY cluster polar cod Boreogadus saida ${ }^{3}$ tRNA pro amplification in CR from Antarctic notthenioig ${ }^{57}$. This research has shown that new tRNAs appear to have been seen in various places. This is the result of rearrangement and mispairing, which ultimately leaves one or more additional tRNAs in the mitochondrial genomes of different fish species. 


\begin{tabular}{|c|c|c|c|c|c|c|c|c|}
\hline \multirow[t]{2}{*}{ Gene } & \multicolumn{2}{|c|}{ Location } & \multirow[t]{2}{*}{ Size } & \multirow[t]{2}{*}{ Anticodon } & \multicolumn{2}{|c|}{ codon } & \multirow[t]{2}{*}{ Intragenic Nucleotides } & \multirow[t]{2}{*}{ Strand } \\
\hline & from & To & & & start & stop & & \\
\hline tRNA Phe & 1 & 68 & 68 & GAA & & & & $\mathrm{H}$ \\
\hline 12SrRNA & 69 & 1081 & 1013 & & & & 0 & $\mathrm{H}$ \\
\hline tRNA val & 1082 & 1151 & 70 & UAC & & & 0 & $\mathrm{H}$ \\
\hline 16SrRNA & 1153 & 2854 & 1702 & & & & +1 & $\mathrm{H}$ \\
\hline tRNA leu & 2855 & 2929 & 75 & UAA & & & 0 & $\mathrm{H}$ \\
\hline ND1 & 2930 & 3904 & 975 & & ATG & TAG & 0 & $\mathrm{H}$ \\
\hline tRNA Ile & 3914 & 3984 & 71 & GAU & & & +9 & $\mathrm{H}$ \\
\hline tRNA GIn & 3984 & 4054 & 71 & UUG & & & -1 & $\mathrm{H}$ \\
\hline tRNA met & 4054 & 4123 & 70 & CAU & & & 0 & L \\
\hline ND2 & 4124 & 5168 & 1045 & & ATG & TAG & 0 & $\mathrm{H}$ \\
\hline tRNA trp & 5169 & 5241 & 73 & UCA & & & 0 & $\mathrm{H}$ \\
\hline tRNA ala & 5243 & 5312 & 70 & UGC & & & +2 & $\mathrm{H}$ \\
\hline tRNA asn & 5314 & 5386 & 73 & GUU & & & 0 & $\mathrm{H}$ \\
\hline $\mathrm{OL}$ & 5387 & 5420 & 34 & & & & 0 & L \\
\hline tRNA cys & 5421 & 5487 & 67 & $\mathrm{GCA}$ & & & 0 & L \\
\hline tRNA tyr & 5488 & 5558 & 71 & GUA & & & 0 & L \\
\hline $\mathrm{COI}$ & 5560 & 7128 & 1569 & & GTG & TAA & +1 & L \\
\hline tRNA ser & 7120 & 7192 & 73 & UGA & & & -7 & $\mathrm{H}$ \\
\hline tRNA Asp & 7198 & 7269 & 72 & GUC & & & +7 & L \\
\hline COII & 7284 & 7974 & 691 & & ATG & $\mathrm{T}$ & +14 & $\mathrm{H}$ \\
\hline tRNA lys & 7975 & 8048 & 74 & UUU & & & 0 & $\mathrm{H}$ \\
\hline ATP8 & 8050 & 8217 & 168 & & ATG & TAA & +1 & $\mathrm{H}$ \\
\hline ATP6 & 8208 & 8891 & 684 & & ATG & TAA & -8 & $\mathrm{H}$ \\
\hline COIII & 8891 & 9675 & 785 & & ATG & TAA & 0 & $\mathrm{H}$ \\
\hline tRNA Gly & 9676 & 9748 & 73 & UCC & & & 0 & $\mathrm{H}$ \\
\hline ND3 & 9749 & 10097 & 349 & & ATG & TAG & 0 & $\mathrm{H}$ \\
\hline tRNA Arg & 10098 & 10167 & 70 & UCG & & & 0 & $\mathrm{H}$ \\
\hline ND4L & 10168 & 10464 & 297 & & ATG & TAA & 0 & $\mathrm{H}$ \\
\hline ND4 & 10458 & 11838 & 1381 & & ATG & $\mathrm{T}$ & -5 & $\mathrm{H}$ \\
\hline tRNA His & 11839 & 11907 & 69 & GUG & & & 0 & $\mathrm{H}$ \\
\hline tRNA ser & 11908 & 11975 & 68 & GCU & & & 0 & $\mathrm{H}$ \\
\hline tRNA leu & 11976 & 12048 & 73 & UAG & & & 0 & $\mathrm{H}$ \\
\hline ND5 & 12049 & 13890 & 1842 & & ATG & TAA & 0 & $\mathrm{H}$ \\
\hline ND6 & 13887 & 14408 & 522 & & ATG & TAG & -2 & L \\
\hline tRNA Glu & 14409 & 14478 & 70 & UUC & & & +4 & L \\
\hline
\end{tabular}




\begin{tabular}{|c|c|c|c|c|c|c|c|c|}
\hline tRNA Glu & 14483 & 14552 & 70 & UUC & & & 0 & $\mathrm{~L}$ \\
\hline cytb & 14555 & 15695 & 1141 & & ATG & $\mathrm{T}$ & +2 & $\mathrm{H}$ \\
\hline tRNA thr & 15696 & 15769 & 74 & UGU & & & 0 & $\mathrm{H}$ \\
\hline tRNA pro & 15773 & 15842 & 70 & UGG & & & +3 & $\mathrm{~L}$ \\
\hline D-loop & 15842 & 16836 & 994 & & & & 0 & - \\
\hline
\end{tabular}

Table 1. Summary of gene feature of Huso huso

\subsection{Protein coding genes}

The cumulative length of H.huso mitochondrial protein coding is $11406 \mathrm{bp}$, which is calculated \% 67 of the total length of the mitochondrial genome.

The genes encoded in mtDNA are highly compact. It also contains overlapping sections. Our overlap was between 13 protein-encoding genes in this species between ATP8 and ATP6, which had 8 bp overlap, and between ND4L and ND4, which was 5 bp, and between ND5 and ND6, that was found to be $2 \mathrm{bp}$.

Most genes encoding mitochondrial proteins in this species begin with the ATG primer, which is similar to that of many metazoas ${ }^{54}$. The COI gene only had one separate START CODON in the form of GTG. Among the coding genes for the protein, the cytb, COII, and ND4 genes terminate in T (Table 1). and this incomplete codon was completed with the addition of poly A and became TAA.

The calculation of open nucleotide abundance at each codon position in all 13 protein genes is shown in the table. T nucleotide was mostly seen in the position of the second codon $(p<0.01)$. Since triple codons are encoded with $T$ in the second position for hydrophobic or hydrophobic residues, this observed deviation indicates the high ratio of hydrophobic residues between the coding proteins. The 12 genes, encoding protein, in the heavy strand share an anti-G deviation. About $10 \%$ or less, $(p<0.01)$ at the third codon position.

The deviation, primarily in the position of the third codon, is probably due to the selection pressure on the synonymous mutation in this position (Table2). 


\begin{tabular}{llllllllllllllllll} 
Genes & Total & & \multicolumn{4}{c}{} & \multicolumn{4}{c}{ First position } & \multicolumn{4}{c}{ Second position } & \multicolumn{4}{c}{ Third position } \\
\hline & A\% & T\% & G\% & C\% & A\% & T\% & G\% & C\% & A\% & T\% & G\% & C\% & A\% & T\% & G\% & C\% \\
\hline ND1 & 29.1 & 25.4 & 13.8 & 31.6 & 24.9 & 21.5 & 25.2 & 28.3 & 18.8 & 40.9 & 11.4 & 28.9 & 43.7 & 13.8 & 4.9 & 37.6 \\
\hline ND2 & 31.7 & 21.9 & 13.4 & 33.0 & 36.4 & 15.5 & 20.6 & 27.5 & 16.7 & 37.4 & 11.5 & 34.5 & 40.0 & 12.9 & 8.0 & 37.1 \\
\hline COI & 24.3 & 27.5 & 19.6 & 28.4 & 24.3 & 22.4 & 31.4 & 22.0 & 18.4 & 40.0 & 15.3 & 26.4 & 30.4 & 20.3 & 12.2 & 36.7 \\
\hline COII & 30.8 & 25.8 & 17.1 & 26.3 & 23.4 & 19.0 & 31.2 & 26.4 & 28.3 & 38.3 & 10.9 & 22.6 & 40.9 & 20.0 & 9.1 & 30.0 \\
\hline ATP8 & 32.7 & 25.0 & 11.9 & 30.4 & 33.9 & 23.2 & 12.5 & 30.4 & 25.0 & 33.9 & 12.5 & 28.6 & 39.3 & 17.9 & 10.7 & 32.1 \\
\hline ATP6 & 26.8 & 25.3 & 13.4 & 34.4 & 28.5 & 13.2 & 21.9 & 36.4 & 14.5 & 47.8 & 11.8 & 25.9 & 37.3 & 14.9 & 7.0 & 40.8 \\
\hline COIII & 26.4 & 25.4 & 17.5 & 30.8 & 20.2 & 23.7 & 29.4 & 26.7 & 21.0 & 36.3 & 16.8 & 26.0 & 37.9 & 16.1 & 6.1 & 39.8 \\
\hline ND3 & 22.9 & 27.8 & 17.8 & 31.5 & 16.2 & 25.6 & 29.9 & 28.2 & 15.5 & 43.1 & 12.9 & 28.4 & 37.1 & 14.7 & 10.3 & 37.9 \\
\hline ND4L & 24.6 & 24.9 & 14.5 & 36.0 & 21.2 & 23.2 & 23.2 & 32.3 & 16.2 & 39.4 & 14.1 & 30.3 & 36.4 & 12.1 & 6.1 & 45.5 \\
\hline ND4 & 28.2 & 24.4 & 15.6 & 31.6 & 29.7 & 18.9 & 21.9 & 29.5 & 16.3 & 40.7 & 14.8 & 28.2 & 38.5 & 13.7 & 10.2 & 37.2 \\
\hline ND5 & 30.7 & 24.2 & 13.0 & 32.1 & 34.7 & 18.9 & 20.5 & 25.9 & 20.4 & 39.4 & 12.1 & 28.2 & 37.0 & 14.3 & 6.4 & 42.3 \\
\hline Cytb & 27.0 & 26.3 & 15.3 & 31.4 & 24.9 & 23.4 & 24.9 & 26.8 & 20.8 & 40.5 & 13.7 & 25.0 & 35.3 & 15.0 & 7.4 & 42.4 \\
\hline ND6 & 11.9 & 41.0 & 35.1 & 12.1 & 9.2 & 34.5 & 45.9 & 10.9 & 13.2 & 20.7 & 46.0 & 20.1 & 13.2 & 42.5 & 39.1 & 5.2
\end{tabular}

Table2. The average base composition in 13 protein coding genes in Huso huso.

Serine and Leucine amino acids showed the highest frequency in the A. persicus by using 6 different codons, while some amino acids used 2 or 4 codons. After them, the amino acids of Alanine, Threonine, Glycine, Proline and Valine showed the highest frequency with four codon usage. Of course, Arginine also had four codons and showed relatively high frequency. The rest of the amino acids used two codons, which showed the same frequency (Figure 2).

\section{4. rRNA and tRNA}

The assessment of rRNAs in this species showed that the lengths were 12 SrRNA 1013bp and 16 srRNA 1702bp. Both rRNAs were encoded by the $\mathrm{H}$ strand and their nucleotide composition was for 12 SrRNA A\% 31.4 C\% 27.5 T\% $19.3 \mathrm{G} \% 21.7$ and for 16 srRNA A\% $35.5 \mathrm{C} \% 24.6 \mathrm{~T} \% 19.6 \mathrm{G} \%$ 20.0. This emphasizes that the deviation of the nucleotide composition is a strand-specific property in mtDNA and this deviation observed is against G. Similar phenomena has been found in the mitochondrial genome of other fishes ${ }^{39}$ and mammals ${ }^{42}$ that are thought to be related to asymmetric replication of $\mathrm{H}$ and $\mathrm{L}$ strand ${ }^{42}$ Especially in the stem 16SrRNA and 12SrRNS, which is necessary for stability in the stem structure, deviation against $\mathrm{G}$ was less ${ }^{52}$. In region $5^{\prime}$ 12SrRNA, a 52-nucleotide VNTR sequence was observed with the following sequence, which could be the result of mispairing:

\section{AGGCTTGGTCCTGGCCTTACTATCAATTTTAACCCAATTTACACATGCAAGT}

This sequence has the ability to produce stems and loops and a stable structure.

The tRNAs, 14 of tRNAs are encoded by the heavy strand and the other 8 by the light strand. This tRNAs are 67 to 75 bp.

All tRNAs except ser (AGY) can be converted to clover leaves, the structure of which can be determined in tRNA-scan. The tRNAser was reduced in the dehydrooridine arm, transforming its second structure into a short clover leaf, which is similar to most metazoa

${ }^{16}$ (Figure 3). 


\subsection{Non-coding regions}

Non-encoded regions in mtDNA include the following: OL and CR and several regions between genes. The size of CR is 994 bp and it is actually downstream tRNAper and is the largest non-coding region. This region is rich in AT.

CR contains several TASs and the TAS sequence extends in several CR regions. This sequence is associated with the CSB conserved sequence blocks. The conserved CSB-1-3 sequence blocks are downstream of this region. The TAS region is rich in repeat and is the most variable part of the CR. It contains duplicate elements and has length $82 \mathrm{of} \mathrm{bp}$. It contains a conserved TACAT motif, and the TAS motif is an inverse complement to ATGTA. The TAS motif can be paired with the CTAS motif, resulting in the formation of stable hair pin loops, which may also serve as a specific sequence signal to terminate mtDNA replication ${ }^{45}$. Repeat sequences occur in this region in fish of different species ${ }^{4-5}$. A comparison of mtDNA sequences in sturgeon and conserved sequences of termination replication (TAS) sturgeons with changes in the number of consecutive duplicate sequences, shows that they can form stable structures during mtDNA replication. In the mtDNA of sturgeons, the control region responsible for the termination of $\mathrm{H}$-strand replication contains one to seven variable number tandem repeats (VNTR) with a unit size of 78 to $83 \mathrm{bp}{ }^{26}$. The D-loop of sturgeon mtDNA is different from human D-loop and contains of more than one TAS; therefore, the termination of mtDNA replication cannot be explained by helicase activity alone. Additionally, it is unclear why sturgeons exhibit a wide range of haplotypes differing in VNTR length and,

correspondingly, in the number of TAS elements. ${ }^{26}$.Kornienko et al. (2019) identified in all sturgeon species studied, VNTR regions contained highly conservative sequences that it terminate with an CAT triplet. TAS elements located in repeating units that constitute VNTR. The TAS nucleotide sequences associated with the termination of mtDNA replication ${ }^{26}$. An ineffective DNA repair system and a lack of protective histones in this organelle causes the mutation rate in mitochondrial DNA to be higher than the DNA nucleus that VNTRs are its result.Tandem repeats in this region increas lengh D-oop and lower D-loop increases the rate of super coil formation ${ }^{46}$. This has effect on protein bonding and more accessibility as well as on transcription and replication ${ }^{44}$.

Variation in repeat tandems in this region probably evolved through the process of illegitimate elongation ${ }^{5}$. It occurs during mtDNA replication and possibly is the mechanism mediated by slipped strand and then mispairning ${ }^{15}$.

We identified conserved CSBD and CSB-1,2,3 sequence blocks with high similarity of CSB sequence from other fishes (Figure 4). While 5 conserved block sequences have been reported in the conserved central domain in mammals in the control region, only 3 of CSBs are typically found in bony fishes ${ }^{19,28,30}$. The CSB-D plays a critical role in maintaining regulatory performance in CR and is considered the most protected part of CR ( $95 \%$ of its sequence is conserved). The CSB 1,2,3 sequences had the least conserving. Consensus sequences for CSB can be found here:

CSB-D, TACTGGCATCTGATTAAT;

CSB-1, TAATAGATAGTGAATGATATAATGACATA;

CSB-2, CAAACCCCCTACCCCC;

CSB-3, TGTCAAACCCCAAAAGCA.

In addition, three conserved sequences were found downstream of the protected central conserved domain, the CSB-1 is an AT-rich region following a GATACA conserved motif.

The downstream CSBs are separated by a poly C and are identified by TTA or TA. CSB 2 consists of a sequence with a polyC stretch. These CSBs appear to be involved in the formation of primer RNA for mtDNA amplification and play a key role in the RNA switch in DNA synthesis, which actually begins in the OL region ${ }^{10,14}$.

The non-coding OL region is located in a cluster of five tRNA genes (trp (W), Ala (A), Asn (N), cys (c) and tyr (y)). It is called the WANCY region. The $\mathrm{OL}$ region has 34 nucleotides and is identified by a stable loop stem structure with a GC-rich stem and a T-rich loop. Two common features of vertebrate OL are the 5'Flanking region rich in primidine (stem) and a motif (5区-CTTCCT-3邓) found in the stem (Figure 5).

Both may be related to the accuracy and efficiency of DNA replication in $\mathrm{OL}$, as seen in the human mitochondrial genome ${ }^{22}$. 


\section{6. Phylogenetic analysis}

Recent phylogenetic analysis based on the mitochondrial genome for 13 protein-encoding genes by not removing the repeat regions, may lead to multiple shifts in a number of site reduced homoplasys, we performed partition maximum likelihood (ML) using the concatenated nucleotide sequences of the 13 Hstrand protein coding genes. We excluded the VNTRs as they are rapidly evolving sequence and it may lead to multiple substitutions at some sites so that high heteroplasy would reduce the resolution of our phylogeny. Their shape was uniformly marked with a bootstrap 1000 on the ML tree. Monophilic species with Huso huso including Acipenser guelenstaedtii, Acipenser stellatus and Acipenser nudiventris were placed in a Clad.

Paraphilic members were grouped in separate clad that includes acipenser sinensis, acipenser schrenckii, acipenser transmontanous and acipenser dabryanous. Our results agree with the taxonomy for sturgeons within the genus and reflected monophilic and paraphilic taxons (Figure 6).

Short mitochondrial gene fragments have revealed limitations in complex phylogenetic relationships in many of our lineages. Many informative sites from longer DNA sequences, such as the complete mitochondrial genome, allow deeper branches and higher levels of relationships to resolve complex relationships. Based on more genetic data the mitochondrial genome will completely elucidate the phylogenetic relationships and determine a higher level of lineage.

We identified the complete mitochondrial genome consisting of 22 tRNAs, 2 rRNAs, 13 protein-encoding genes, and two OL and D-loop control regions. The VNTR is found in three sites and also a VNTR is between ND6 and Cytb as pseudo tRNA-Glu. This organization of the genome was separate from other previously reported sturgeon species. This included an increase in a tRNA in H.huso and a VNTR in the 12 srRNA region.

Looking into more details showed that the divergence between substitution in the mitochondrial genome of rRNA and tRNA was less than the coding genes of the protein, indicating the stability of the stem and loop in the rRNA and tRNA. The CR had the most divergence. The divergence ratio varies between the genes encoding the protein and ATP8, ND3, ND6, Cytb and ND4L showing the highest conservation, but ND2 and CO2 showing the highest divergence. ND2 is a small protein encoding gene that is amplified by a primer and can be used as a protein encoding gene with good divergence in barcoding species, which is much more suitable than Col (The complete gene can be used instead of the partial gene). Applying these new genomic sequences to taxonomic tests of this specie will be very useful and will have achievements for phylogenetic analysis and the study of lineage rearrangements, conservation and evaluation of biological studies.

We calculated the ratio of non-synonymous substitutions (Ka) to the rate of synonymous substitution (Ks) (Figure7). Our results show that all 13 genes have a Ka / Ks ratio lower than one, indicating a strong selection signal for harmful mutations in all mitochondrial protein genes. However, the mean Ka / Ks ratio (0.1to/0.001) shows a significant difference between individual genes. The highest rate (ND2, COX2) indicates that the purification selection is under minimal pressure.

\section{Conclusion}

The 13 genes encoding proteins show that they are less conserved than rRNA and tRNA. Divergence varies between genes. COII were the highest, while the AT8 synthetase subunit was the lowest. Observing the divergence rates of these genes allows us to compare them for barcoding. Although Col is commonly used for DNA barcoding, but low divergence has been observed in this gene, indicating that it's not able to clearly distinguish very close species. The data analyze has shown that ND2 is a better candidate for barcode identification in grouping. It has higher percentage of variable sites than COI. Therefor it can differentiate between newly derived species.

In addition, the smaller size of ND2 makes it easier to use compared to Col. The sequence is completely amplified using only one pair of primer. Thus a complete gene instead of a partial gene can be used for barcoding.

Nevertheless, we observed a kind of diversity in this particular species, which include the presence of tandem repeat in the control region and 12SrRNA and tRNA-Glu, which is the result of mispairing. So far, there have been reports of pseudo tRNA in several species of fish, but there have been no reports about the presence of repeats in fish, in 12srRNA. 


\section{Declarations}

\section{Acknowledgements}

Thanks to the experts from the Caspian Sea Ecology Institute in Sari, who have been involved in the sampling of sturgeon fish for this research.

\section{References}

1. Barmintseva, A. E. \& Mugue, N. S. Genetic Variation of the Siberian Sturgeon (Acipenser baerii Brandt, 1869) in Aquaculture. Russian journal of genetics, 54 (2), 210-217 (2018).

2. Boscari, E. et al. Species and hybrid identification of sturgeon caviar: a new molecular approach to detect illegal trade. Molecular ecology resources, 14 (3), 489-498 (2014).

3. Breines, R., Ursvik, A., Nymark, M., Johansen, S. D. \& Coucheron, D. H. Complete mitochondrial genome sequences of the Arctic Ocean codfishes Arctogadus glacialis and Boreogadus saida reveal oriL and tRNA gene duplications. Polar Biol, 31 (10), 12451252 (2008).

4. Broughton, R. E. \& Dowling, T. E. (1997) Evolutionary dynamics of tandem repeats in the mitochondrial DNA control region of the minnow Cyprinella spiloptera. Mol Biol Evol 14: 1187-1196. doi:10.1093/ oxfordjournals.molbev.a025728. PubMed: 9402730.

5. Buroker, N. E. et al. Length heteroplasmy of sturgeon mitochondrial DNA: an illegitimate elongation model., 124 (1), 157-163 (1990).

6. Cantatore, P., Gadaleta, M. N., Roberti, M., Saccone, C. \& Wilson, A. C. Duplication and remoulding of tRNA genes during the evolutionary rearrangement of mitochondrial genomes. Nature, 329 (6142), 853-855 (1987).

7. Çiftci, Y., Eroğlu, O. \& Firidin, Å. 2013. Heteroplasmy and length variation in the tRNApro-Dloop regions of three sturgeon species (A. stellatus, A. gueldenstaedtii and H. huso) from the Turkish coast of the Black Sea.Turkish Journal of Biochemistry/Turk Biyokimya Dergisi, 38(3).

8. Çiftci, Y., Eroğlu, O. \& Firidin, Å. Mitochondrial cytochrome b sequence variation in three Sturgeon species (A. stellatus Pallas, 1771, A. gueldenstaedtii Brandt, 1833, H. huso Linnaeus, 1758) from the Black Sea Coasts of Turkey. Turkish Journal of Fisheries and Aquatic Sciences, 13 (2), 291-303 (2013).

9. Clayton, D. A. Replication of animal mitochondrial DNA., 28 (4), 693-705 (1982).

10. Clayton, D. A. "Nuclear gadgets in mitochondrial DNA replication and transcription.". Trends in biochemical sciences, 16, 107-111 (1991).

11. Congiu, L., Fontana, F., Patarnello, T., Rossi, R. \& Zane, L. The use of AFLP in sturgeon identification. Journal of Applied Ichthyology, 18 (4-6), 286-289 (2002).

12. Cui, Z., Liu, Y., Li, C. P. \& Chu, K. H. Species delineation in Pampus (Perciformes) and the phylogenetic status of the Stromateoidei based on mitogenomics. Molecular biology reports, 38 (2), 1103-1114 (2011).

13. Dugo, M. A. et al. Conservation and management implications of fine-scale genetic structure of Gulf sturgeon in the Pascagoula River, Mississippi. Journal of Applied Ichthyology, 20 (4), 243-251 https://doi.org/10.1111/j.1439-0426.2004.00572.x (2004).

14. Doersen, C. J., Guerrier-Takada, C., Altman, S. \& Attardi, G. Characterization of an RNase P activity from HeLa cell mitochondria. Comparison with the cytosol RNase P activity. Journal of Biological Chemistry, 260 (10), 5942-5949 (1985).

15. Fumagalli, L., Taberlet, P., Favre, L. \& Hausser, J. Origin and evolution of homologous repeated sequences in the mitochondrial DNA control region of shrews. Molecular biology and evolution, 13 (1), 31-46 (1996).

16. Garey, J. R. \& Wolstenholme, D. R. Platyhelminth mitochondrial DNA: evidence for early evolutionary origin of a tRNA ser AGN that contains a dihydrouridine arm replacement loop, and of serine-specifying AGA and AGG codons. Journal of Molecular Evolution, 28 (5), 374-387 (1989).

17. Georgescu, S. E. et al. Microsatellite variation in Russian sturgeon (Acipenser gueldenstaedtii) from aquaculture. Scientific Papers Animal Science and Biotechnologies, 47 (1), 73-76 (2014).

18. Georgescu, S., Canareica, O., Dudu, A. \& Costache, M. Analysis of the Microsatellite Variation in the Common Hybrid Between Russian Sturgeon (Acipenser Gueldenstaedtii Brandt and Ratzeburg, 1833) and Siberian Sturgeon (Acipenser Baerii Brandt, 1869) from Aquaculture. Transylvanian Review of Systematical and Ecological Research, 15 (2), 117-124 (2013).

Page 10/19 
19. Guo, X., Liu, S. \& Liu, Y. Comparative analysis of the mitochondrial DNA control region in cyprinids with different ploidy level., 224 $(1-4), 25-38$ (2003).

20. Hassanin, A., LéGer, N. \& Deutsch, J. (2005) Evidence for Multiple Reversals of Asymmetric Mutational Constraints during the Evolution of the Mitochondrial Genome of Metazoa, and Consequences for Phylogenetic Inferences. Syst Biol 54: 277-298. doi: 10.1080/10635150590947843. PubMed: 16021696.

21. Hall, T. A. 1999, January. BioEdit: a user-friendly biological sequence alignment editor and analysis program for Windows 95/98/NT. In Nucleic acids symposium series (Vol. 41, No. 41, pp. 95-98). [London]: Information Retrieval Ltd., c1979-c2000.

22. Hixson, J. E., Wong, T. W. \& Clayton, D. A. Both the conserved stem-loop and divergent 5'-flanking sequences are required for initiation at the human mitochondrial origin of light-strand DNA replication. Journal of Biological Chemistry, 261 (5), 2384-2390 (1986).

23. Inoue, J. G., Miya, M., Tsukamoto, K. \& Nishida, M. Evolution of the deep-sea gulper eel mitochondrial genomes: large-scale gene rearrangements originated within the eels. Molecular biology and evolution, 20 (11), 1917-1924 (2003).

24. Jacobs, H. T. et al. Conserved tRNA gene cluster in starfish mitochondrial DNA. Current genetics, 15 (3), 193-206 (1989).

25. Kong, X. et al. A novel rearrangement in the mitochondrial genome of tongue sole, Cynoglossus semilaevis: control region translocation and a tRNA gene inversion. Genome, 52 (12), 975-984 (2009).

26. Kornienko, I. V. et al. "Termination of Replication and Mechanisms of Heteroplasmy in Sturgeon Mitochondrial DNA." Molecular Biology, 53 (no. 1), 107-117 (2019).

27. Ladoukakis, E. D. \& Zouros, E. Evolution and inheritance of animal mitochondrial DNA: rules and exceptions. Journal of Biological Research-Thessaloniki, 24 (1), 1-7 (2017).

28. Lee, W. J., Conroy, J., Howell, W. H. \& Kocher, T. D. Structure and evolution of teleost mitochondrial control regions. Journal of Molecular Evolution, 41 (1), 54-66 (1995).

29. Ludwig, A., May, B., Debus, L. \& Jenneckens, I. Heteroplasmy in the mtDNA control region of sturgeon (Acipenser, Huso and Scaphirhynchus)., 156 (4), 1933-1947 (2000).

30. Liu, M., Li, J. L., Ding, S. X. \& Liu, Z. Q. Epinephelus moara: a valid species of the family Epinephelidae (Pisces: Perciformes). Journal of fish biology, 82 (5), 1684-1699 (2013).

31. May, B., Krueger, C. C. \& Kincaid, H. L. Genetic variation at microsatellite loci in sturgeon: primer sequence homology in Acipenser and Scaphirhynchus. Canadian Journal of Fisheries and Aquatic Sciences, 54 (7), 1542-1547 (1997).

32. Mabuchi, K., Miya, M., Satoh, T. P., Westneat, M. W. \& Nishida, M. Gene rearrangements and evolution of tRNA pseudogenes in the mitochondrial genome of the parrotfish (Teleostei: Perciformes: Scaridae). Journal of Molecular Evolution, 59 (3), 287-297 (2004).

33. MCQuown, E. C., Sloss, B. L. \& Sheehen, R. J. 2000.Microsatellite analysis of genetic variation in sturgeon. New primer sequences for Scaphyhinchus and Acipenser.transactions of the American fisheries society.129:1380.1388. doi.org/10.1577/1548-8659.

34. Miya, M. \& Nishida, M. Organization of the mitochondrial genome of a deep-sea fish, Gonostoma gracile (Teleostei: Stomiiformes): first example of transfer RNA gene rearrangements in bony fishes. Mar. Biotechnol, 1 (5), 416-426 (1999).

35. Moghim, M., Tan, S. G., Javanmard, A., Pourkazemi, M. \& Malar, J. Inheritance of microsatellite loci and their application for pedigree analysis in the polyploid Persian sturgeon (Acipenser persicus). Zoological Studies, 51 (8), 1507-1514 (2012).

36. Mugue, N. S., Barmintseva, A. E., Rastorguev, S. M., Mugue, V. N. \& Barmintsev, V. A. Polymorphism of the mitochondrial DNA control region in eight sturgeon species and development of a system for DNA-based species identification. Russian Journal of Genetics, 44 (7), 793-798 (2008).

37. Mugue, N., Barmintseva, A., Schepetov, D., Shalgimbayeva, G. \& Isbekov, K. Complete mitochondrial genomes of the critically endangered Ship sturgeon Acipenser nudiventris from two seas. Mitochondrial DNA Part B, 1 (1), 195-197 (2016).

38. Ogden, R. et al. Sturgeon conservation genomics: SNP discovery and validation using RAD sequencing. Molecular ecology, 22 (11), 3112-3123 (2013).

39. Ortí, G., Petry, P., Porto, J. I., Jégu, M. \& Meyer, A. Patterns of nucleotide change in mitochondrial ribosomal RNA genes and the phylogeny of piranhas. Journal of Molecular Evolution, 42 (2), 169-182 (1996).

40. Perna, N. T. \& Kocher, T. D. Patterns of nucleotide composition at fourfold degenerate sites of animal mitochondrial genomes. Journal of molecular evolution, 41 (3), 353-358 (1995). 
41. Pourkazemi, M., Skibinski, D. F., Beardmore, A. \& J Application of mtDNA d-loop region for the study of Russian sturgeon population structure from Iranian coastline of the Caspian Sea. Journal of Applied Ichthyology, 15 (4-5), 23-28 (1999).

42. Reyes, A., Gissi, C., Pesole, G. \& Saccone, C. Asymmetrical directional mutation pressure in the mitochondrial genome of mammals. Molecular Biology and Evolution, 15 (8), 957-966 (1998).

43. Raymakers, C. \& Hoover, C. Acipenseriformes: CITES implementation from Range States to consumer countries. Journal of Applied Ichthyology, 18 (4-6), 629-638 https://doi.org/10.1046/j.1439-0426.2002.00398.x (2002).

44. Ruhanen, $\mathrm{H}$. et al. Mitochondrial single-stranded DNA binding protein is required for maintenance of mitochondrial DNA and 7S DNA but is not required for mitochondrial nucleoid organisation. Biochimica et Biophysica Acta (BBA)-Molecular Cell Research, 1803 (8), 931-939 (2010).

45. Saccone, C., Pesole, G. \& Sbisa, E. The main regulatory region of mammalian mitochondrial DNA: structure-function model and evolutionary pattern. Journal of Molecular Evolution, 33 (1), 83-91 (1991).

46. Saitoh, K. et al. Complete nucleotide sequence of Japanese flounder (Paralichthys olivaceus) mitochondrial genome: structural properties and cue for resolving teleostean relationship. Journal of Heredity, 91 (4), 271-278 (2000).

47. Shao, W. et al. Universal DNA primers for amplification complete mitochondrial genome for sturgeons. Conservation Genetics Resources, 6 (2), 305-307 (2014).

48. Shoffner, J. M. (1995). Oxidative phosphorylation disease. The metabolic and molecular bases of inherited disease Vol.1.New york: McGraw-Hill (7TH ED.):PP.1535-1609.

49. Southern, Å. O., Southern, P. J. \& Dizon, A. E. Molecular characterization of a cloned dolphin mitochondrial genome. Journal of molecular evolution, 28 (1-2), 32-42 (1988).

50. Tanaka, M. \& Ozawa, T. Strand asymmetry in human mitochondrial DNA mutations., 22 (2), 327-335 (1994).

51. Timoshkina, N. N., Barmintseva, A. E., Usatov, A. V. \& Mugue, N. S. Intraspecific genetic polymorphism of Russian sturgeon Acipenser gueldenstaedtii. Russian Journal of Genetics, 45 (9), 1098-1106 (2009).

52. Wang, H. Y. \& Lee, S. C. Secondary structure of mitochondrial 12S rRNA among fish and its phylogenetic applications. Molecular Biology and Evolution, 19 (2), 138-148 (2002).

53. Williams, E. P., Peer, A. C., Miller, T. J., Secor, D. H. \& Place, A. R. A phylogeny of the temperate seabasses (Moronidae) characterized by a translocation of the mt-nd6 gene. Journal of Fish Biology, 80 (1), 110-130 (2012).

54. Wolstenholme, D. R. \& Jeon, K. W. A survey of cell biology. Int Rev Cytol, 141, 173-232 (1992).

55. Xiao, T. Q. et al. An AFLP-based approach for the identification of sex-linked markers in A mur sturgeon A cipenser schrenckii B randt, 1869. Journal of Applied Ichthyology, 30 (6), 1282-1285 (2014).

56. Yarmohammadi, M., Pourkazemi, M., Ghasemi, A., HASANZADEH, S. M. \& Chakmehdouz, F. 2011. AFLP reveals no sex-specific markers in Persian sturgeon (Acipenser persicus) or beluga sturgeon (Huso huso) from the southern Caspian Sea, Iran.

57. Zhuang, X. \& Cheng, C. H. (2010) ND6 gene "lost" and found: evolution of mitochondrial gene rearrangement in Antarctic notothenioids. Mol Biol Evol 27: 1391-1403. doi:10.1093/molbev/msq026. PubMed: 20106908

58. Zhuang, X., Qu, M., Zhang, X. \& Ding, S. A comprehensive description and evolutionary analysis of 22 grouper (Perciformes, Epinephelidae) mitochondrial genomes with emphasis on two novel genome organizations. PLoS One, 8 (8), 73561 (2013).

\section{Figures}




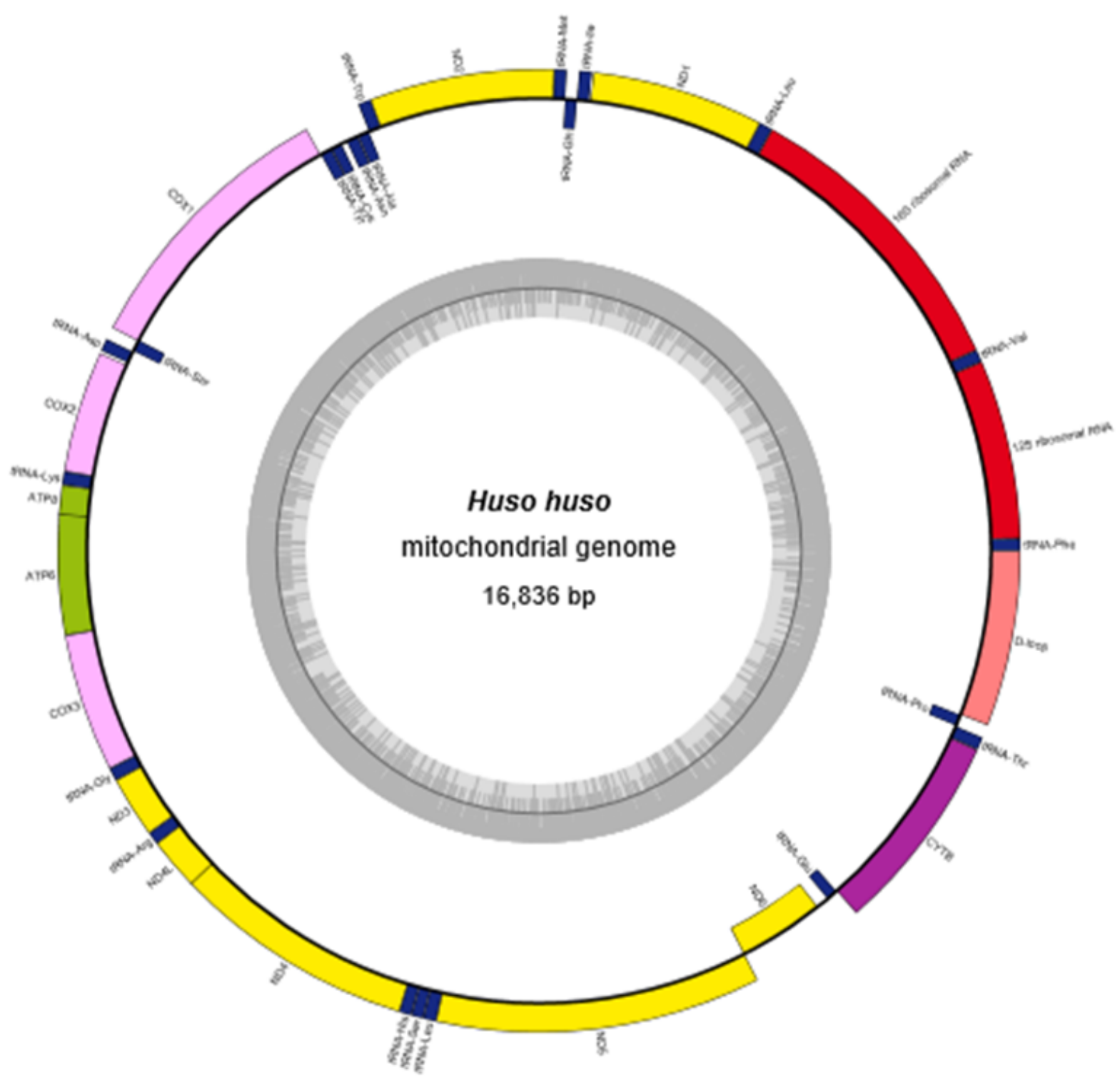

Figure 1

Gene map of the complete mitochondrial genomes along with GC contents for Huso huso 


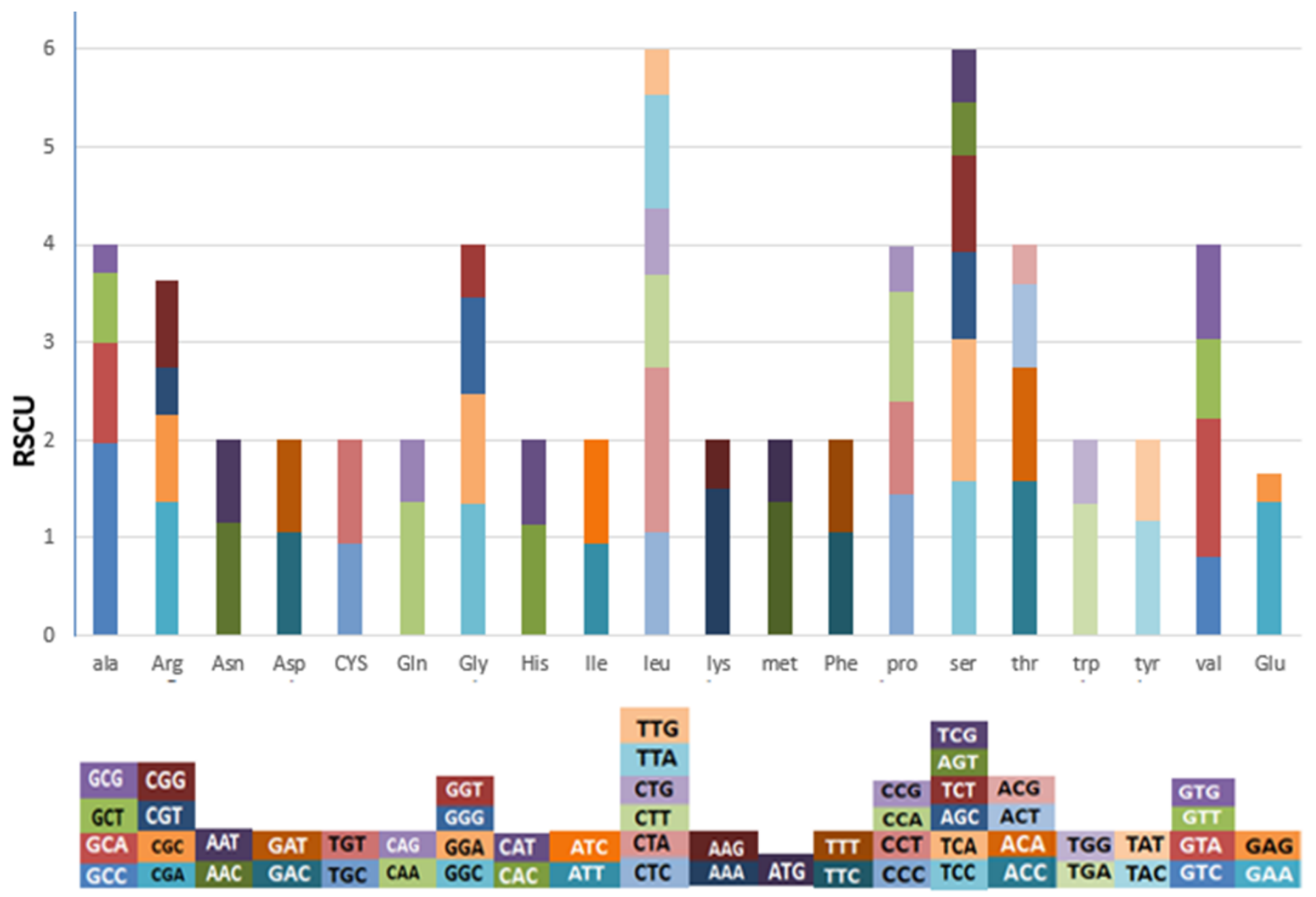

Figure 2

The Relative Synonymous Codon Usage (RSCU) of the mitochondrial protein-coding genes and codon usage of Huso huso for whole genome sequence. 


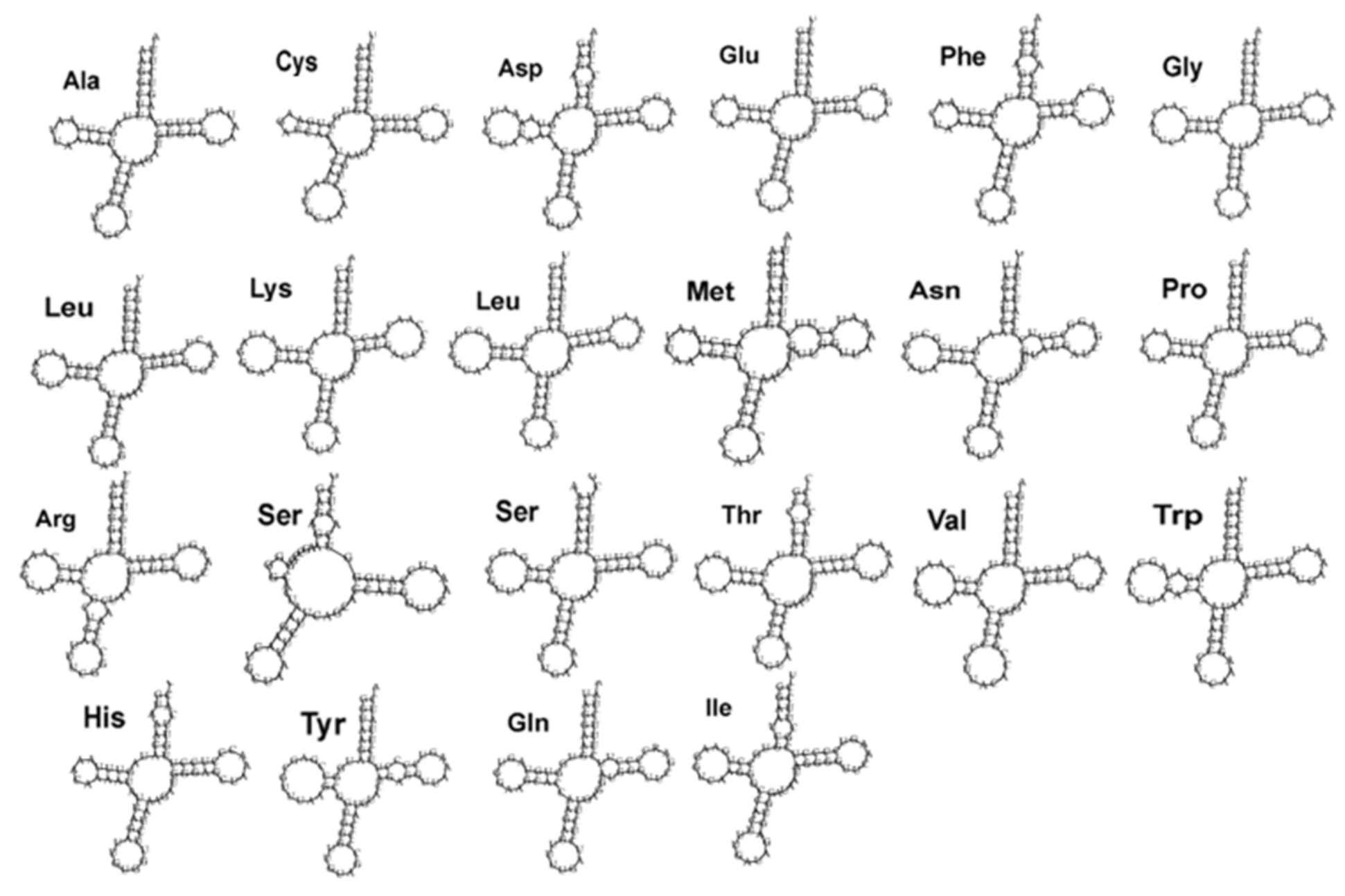

Figure 3

The secondary structure of tRNA genes in Huso huso. 
TAS

trnaP15842ACCACACCATGTTTAACCCACACCAATTTCTAGCCACCATACCACAATGTTTACATATACATTAAATT

ATTCAAGTACATAAGACATGCTATGTATAATCCACATTAATTTCTAGCCACCATACCACAATGTTTACATATACAT

TAAATTATTCAAGTACATAAGACATGCTATGTATAATCCACATTAATTTCTAGCCACCATACCACAATGTTTACAT ATACATTAAATTATTCAAGTACATAAGACATGCTATGTATAATCCACATTAATTTCTAGCCACCATACCACAATGT

TTCATCTACCATTGAATGGTGTACACCATTTTCTCTATGTGTACTAACATGCCCCTTCCTAAAA)CCATAACATGTAG TAAGAGCCGAACATCCTTGTCTGTCTAGAACATGAAGTTAATGAGATGAAGGACAATAACCGTGAGATTTCATA ACTGAATTATTACTAACATCTGGCTCCTATTTCAAGTCCATCAACAGTTATTTCCCCATAATAAGTTACTACTGGC CSB-D

ATCTGATTAATGTTAGAGGTACCATAAACTCATGACCCCACATGCCGAGAACCCCACCAACATTTGGTTACTCTT ATTTCGGTTCCCATTCATTGACATGCAAAGCTCCTTCAGAAAAGATTAATAAGGTGGAACATTCAACAGCCTGCT CSB-1

CGCAGGTAATAGATAGTGAATGATATAATGACATATCCTGGACGCCATACATGACCCTGTGCCACGCACATAAG GAGTGTTTCACGTAGACCCAGTCTTTCCCCCCACAATAATAACCAAGATACAAATAAACGTTTATTATCGACAAA CSB-3
CCCCCTACCCCCTTACGCCGGACAGGCCTTATATTTCTIGTCAAACCCCAAAAGCAGGACTGACTTGTCATCAACG TACTCTAATTACCCACATGCGCCTAGTTGCGCAAATGTTTACTCACTGTATTTTIATGTATATACATTATTACACAA TCACACAAAATAATATATAtrnaF

Figure 4

The D-loop region along with the CSB-conserved blocks. 


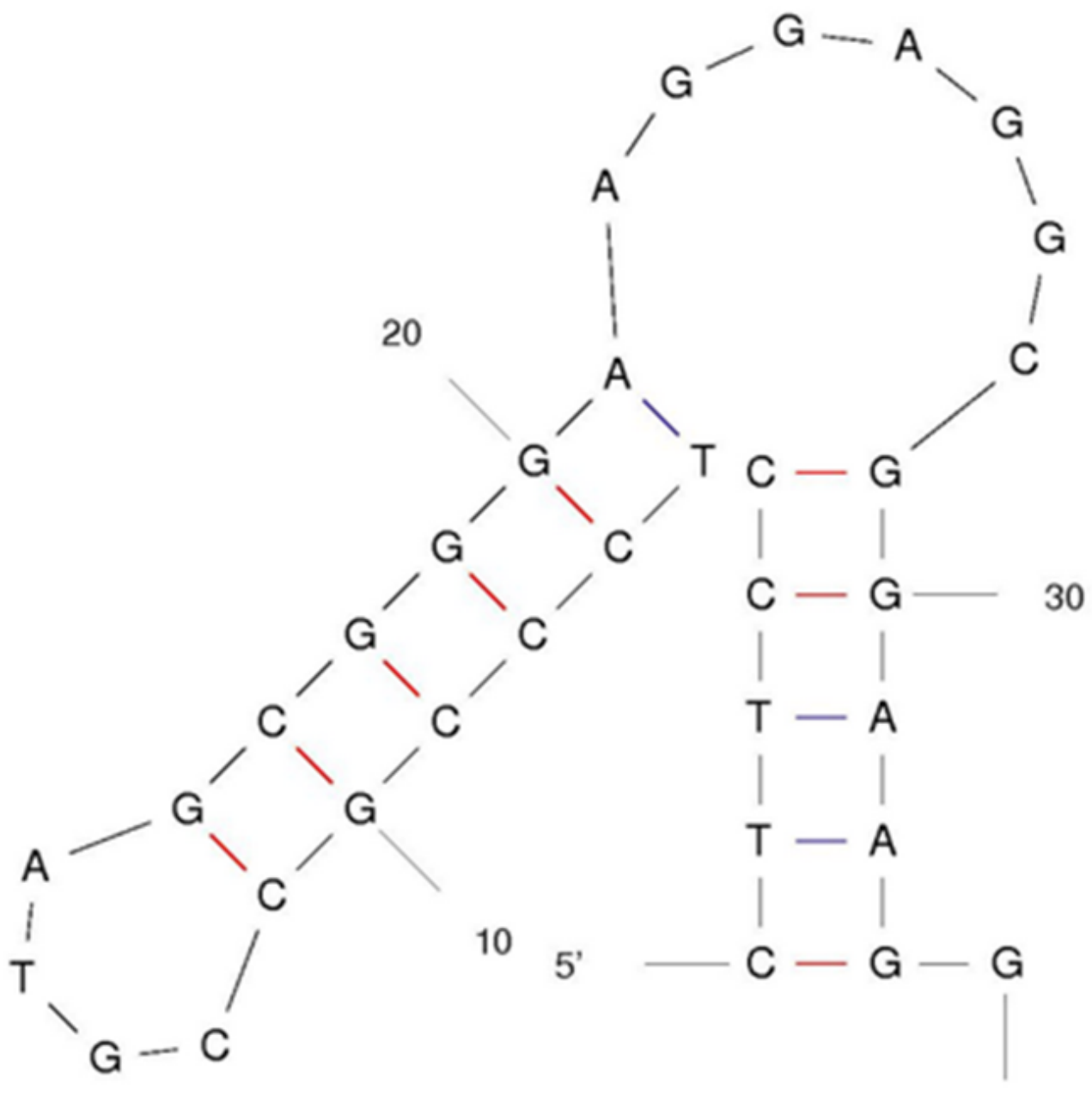

3'

Figure 5

The OL region was found in Huso huso. 


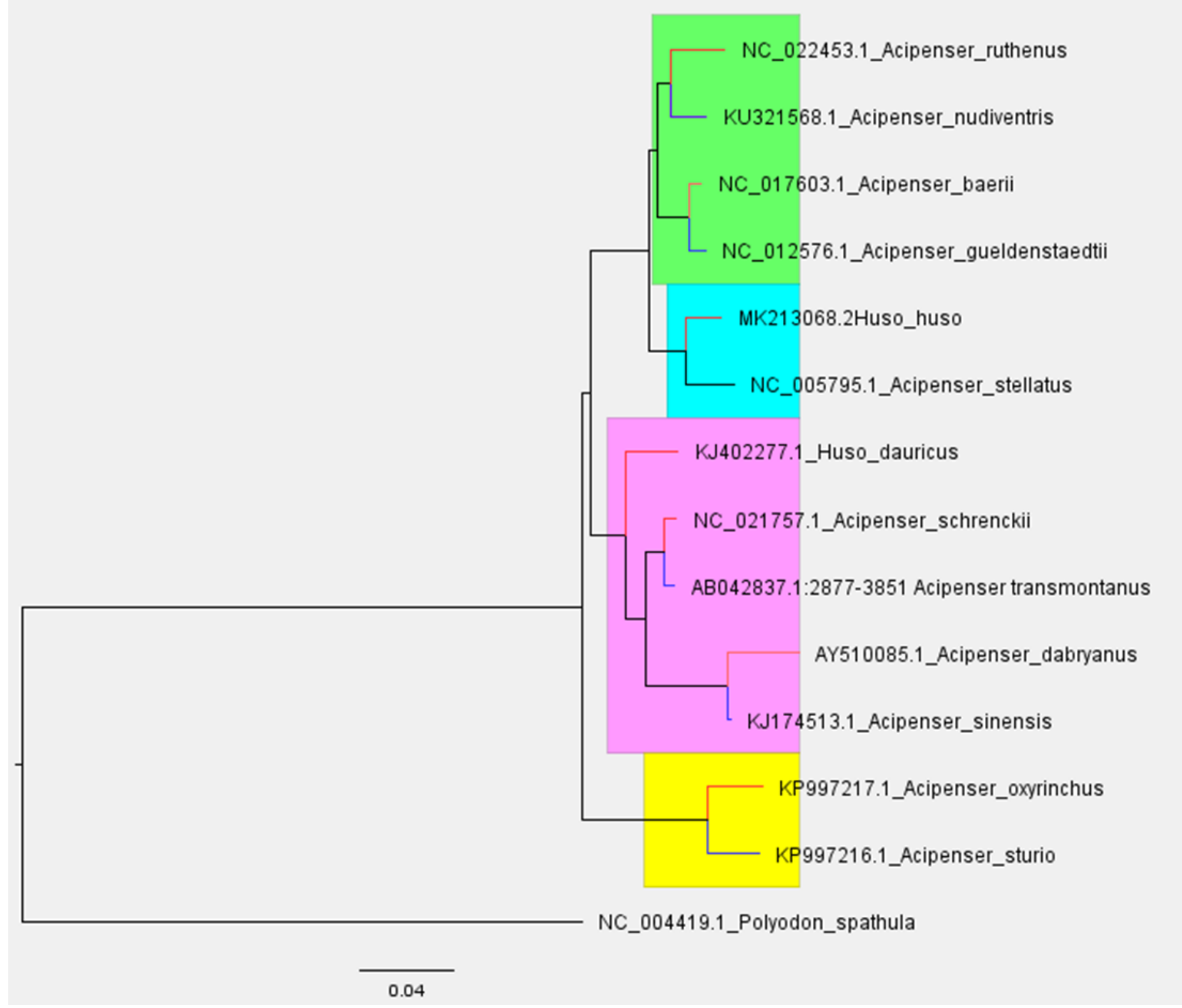

Figure 6

Evolutionary relationship between Huso huso and other sturgeon species by phylogenetic tree. 
$\mathrm{Ka} / \mathrm{Ks}$

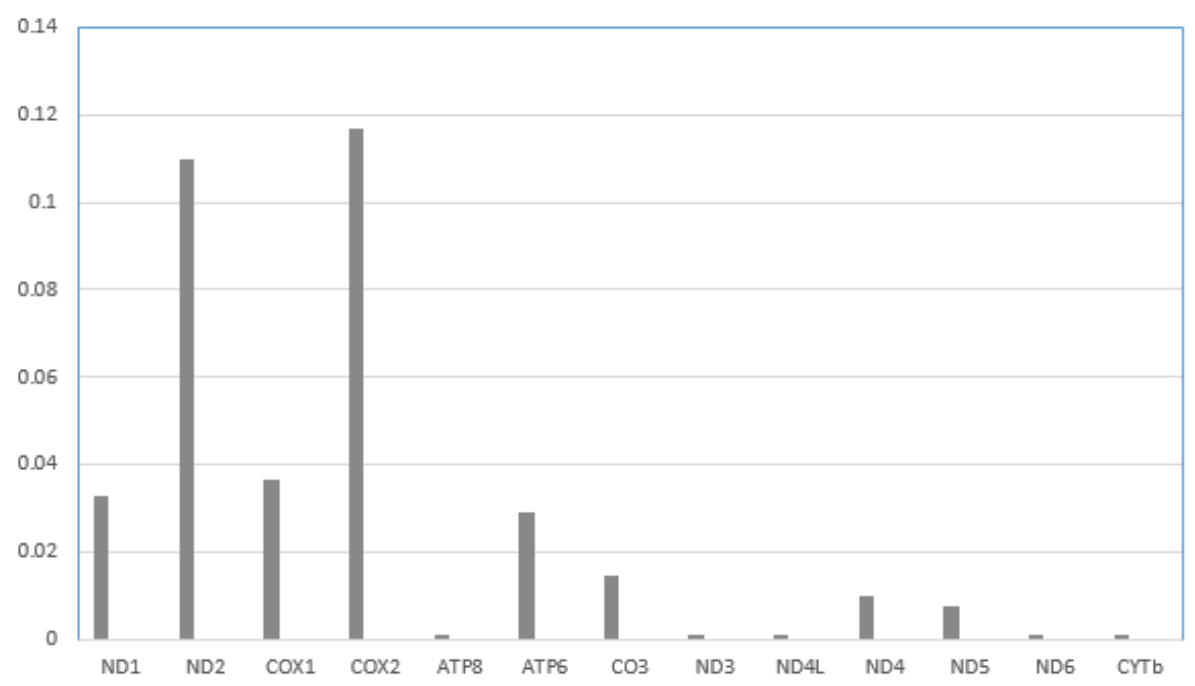

Figure 7

Average Ka /Ks ratios of 13 protein-coding genes. Ka /Ks is the ratio of non-synonymous substitutions rate (Ka) to synonymous substitutions substitution rate (Ks). 